\title{
PRICING MULTI-ASSET OPTIONS WITH AN EXTERNAL BARRIER
}

\author{
YUE-KUEN KWOK, LIXIN WU and HONG YU \\ Department of Mathematics, Hong Kong University of Science and Technology, \\ Clear Water Bay, Hong Kong \\ E-mail:maykwok@usthk.ust.hk
}

Received 19 December 1997

Revised 24 June 1998

\begin{abstract}
An external barrier of an option contract is a stochastic variable which determines whether the option is knocked in or out when the value of the variable is above or below some predetermined level, but itself is not the price of an asset which underlies the option. In this paper, we present analytic formulation for the valuation of European options on one or multiple assets with single external barrier, where the barrier level can be exponential. As the domain of the problem becomes semi-infinite due to the presence of the external barrier, we employ the method of images to find the Green function of the governing differential equation. An efficient and accurate fractional step finite difference scheme is proposed for the numerical valuation of these barrier options.
\end{abstract}

Keywords: Multi-asset options, external barrier, Green's function, fractional step finite difference schemes.

JEL classification code: G130

\section{Introduction}

Heynen and Kat [1] have presented analytic valuation formulas for Europeanstyle barrier options with single external barrier, where the external variable is not the price of the underlying asset. For example, the underlying asset may be a foreign stock and the external barrier may be the exchange rate. Such barrier options become nullified (out-options) or activated (in-options) when the value of the barrier variable hits some level either from above (down-options) or below (up-options). In this paper, we go beyond the scope of Heynen and Kat's formulas by allowing more than one underlying asset and the barrier level can be exponential. In the valuation of these options, we take the usual Black-Scholes assumptions. The diffusion processes for the prices of the assets which underly the option and the barrier variable are taken to be lognormal, and the correlation coefficients between these diffusion processes are all constant. Also, the volatility values and the riskless interest rate are assumed to be constant. Besides the presentation of analytic formulations, an 
efficient implicit finite difference scheme is also proposed for the numerical valuation of these barrier options.

This paper is organized as follows. In the next section, we present the valuation formulas for European-style options on one or multiple assets with single external barrier where the barrier level can be exponential. The transition density function of the underlying processes for the asset prices associated with the barrier feature is derived using the partial differential equation approach rather than the usual statistical approach. We solve for the Green function of the governing differential equation argumented with the homogeneous Dirichlet boundary condition corresponding to the absorbing barrier. The Green function can be recognized to be the discounted value of the transition density function. Since the domain of the problem becomes semi-infinite with the presence of the barrier, we derive the Green function using the method of images. The method of images resembles the reflection principle in statistics [5]. In the present context, we believe that the analytic procedures involved using the method of images are more routine and direct. It is known that the binomial method requires some judicious modifications to achieve sufficient accuracy for the numerical valuation of barrier options [6]. In Sec. 3, we propose a class of fractional step implicit finite difference schemes for the accurate valuation of these barrier options. The pricing behaviors of a European option on the maximum of two risky assets with an external barrier are also investigated. In particular, we analyze the effects of correlations between the diffusion processes on the option price. The paper is ended with conclusive remarks in Sec. 4.

\section{European Options with Single External Barrier}

Suppose there is an external barrier variable which determines whether the option is nullified ("out") or activated ("in") when the value of the barrier variable hits some prescribed level. For European-style options, an in-option plus an outoption with the same expiration date, strike price and barrier will have the same terminal payoff as the corresponding ordinary option without the barrier. Therefore, it becomes necessary only to find values for out-options since those for in-options can be obtained without much added efforts.

Let $B$ denote the external barrier variable, and $b(\tau)=b_{0} e^{-\beta \tau}, \beta>0$, be the exponential barrier level for $B$. The barrier variable is said to be an up (or down) barrier if the out-option becomes nullified when the value of the barrier variable hits the corresponding barrier level from below (or above), where the barrier level is upstream (or downstream) of the movement of the value taken by the barrier variable.

\subsection{Formulation of the Green function}

Assume that there are $m$ underlying assets and let $S_{i}, i=1,2, \ldots, m$, denote the price of the underlying asset $i$. In the risk neutral world, the underlying asset 
prices are assumed to follow the lognormal diffusion processes:

$$
\frac{d S_{i}}{S_{i}}=\left(r-q_{i}\right) d t+\sigma_{i} d Z_{i}, \quad i=1,2, \ldots, m,
$$

where $r$ is the constant riskless interest rate and $\sigma_{i}$ is the constant volatility of the stochastic process for $S_{i}, q_{i}$ is the constant dividend yield of asset $i$. We let $n=m+1$. The value of the barrier variable is also assumed to follow the lognormal process:

$$
\frac{d B}{B}=(r-q) d t+\sigma_{n} d Z_{n}
$$

where $q$ is the dividend yield associated with $B$ and $\sigma_{n}$ is the constant volatility of the stochastic process for $B$. Here, $d Z_{i}, i=1,2, \ldots, n$, are standard Wiener processes, and let $\rho_{i j}$ denote the correlation coefficient between $d Z_{i}$ and $d Z_{j}$.

Suppose we apply the following transformation of variables:

$$
x_{i}=\frac{1}{\sigma_{i}} \ln S_{i}, i=1,2, \ldots, m, \quad \text { and } \quad x_{n}=\frac{1}{\sigma_{n}} \ln \frac{B}{b(\tau)},
$$

the governing partial differential equation for the value of an option on the $m$ underlying assets and with the downstream external barrier is given by

$$
\begin{aligned}
\frac{\partial V}{\partial \tau}= & \frac{1}{2} \sum_{i=1}^{n} \sum_{j=1}^{n} \rho_{i j} \frac{\partial^{2} V}{\partial x_{i} \partial x_{j}}+\sum_{i=1}^{m} \frac{r-q_{i}-\frac{\sigma_{i}^{2}}{2}}{\sigma_{i}} \frac{\partial V}{\partial x_{i}}+\frac{r-q_{n}-\frac{\sigma_{n}^{2}}{2}}{\sigma_{n}} \frac{\partial V}{\partial x_{n}}-r V, \\
& -\infty<x_{i}<\infty, i=1,2, \ldots, m, \quad 0<x_{n}<\infty, \tau>0,
\end{aligned}
$$

where $q_{n}=q+\beta$. (Note: the interval of definition for $x_{n}$ becomes $-\infty<x_{n}<$ 0 when the barrier is upstream.) We would like to find the Green function for the above differential equation which satisfies the homogeneous Dirichlet boundary condition at $x_{n}=0$ and the vanishing far field boundary conditions at $x_{i} \rightarrow \infty, i=$ $1,2, \ldots, n$ and $x_{i} \rightarrow-\infty, i=1,2, \ldots, m$. First, we find the fundamental solution for the above differential equation in the infinite domain: $-\infty<x_{1}, x_{2}, \ldots, x_{n}<\infty$; then use the method of images to find the corresponding Green function in the semi-infinite domain: $-\infty<x_{1}, \ldots, x_{m}<\infty, 0<x_{n}<\infty$. The fundamental solution in the infinite domain to (4) is found to be (see Appendix A)

$$
\begin{aligned}
g_{n}\left(x_{1}, x_{2}, \ldots, x_{n}\right)= & \frac{e^{-r \tau}}{(2 \pi \tau)^{n / 2}} \frac{1}{\sqrt{\operatorname{det} R}} \exp \left(-\frac{1}{2 \tau} \mathbf{y}^{T} R^{-1} \mathbf{y}\right), \\
& -\infty<x_{1}, x_{2}, \ldots, x_{n}<\infty .
\end{aligned}
$$

Here, $R$ is an $n \times n$ symmetric matrix whose entries are $R_{i j}=\rho_{i j}, i, j=1,2, \ldots, n$, and $\mathbf{y}=\left(\begin{array}{llll}y_{1} & y_{2} & \ldots & y_{n}\end{array}\right)^{T}$ where

$$
y_{i}=x_{i}+\mu_{i} \tau, \quad i=1,2, \ldots, n,
$$

where

$$
\mu_{i}=\frac{r-q_{i}-\frac{\sigma_{i}^{2}}{2}}{\sigma_{i}}, \quad i=1,2, \ldots, n
$$


Also, we write $\boldsymbol{\xi}=\left(\begin{array}{llll}\xi_{1} & \xi_{2} \ldots \xi_{n}\end{array}\right)^{T}$ and $\boldsymbol{\eta}=\left(\eta_{1} \eta_{2} \ldots \eta_{n}\right)^{T}$. Corresponding to the homogeneous boundary condition at $x_{n}=0$, the Green function for Eq. (4) defined in the semi-infinite domain can be expressed as

$$
\begin{aligned}
G_{n}(\mathbf{y}, \tau-u ; \boldsymbol{\xi})= & \frac{e^{-r(\tau-u)}}{[2 \pi(\tau-u)]^{n / 2}} \frac{1}{\sqrt{\operatorname{det} R}}\left[\exp \left(-\frac{1}{2(\tau-u)}(\mathbf{y}-\boldsymbol{\xi})^{T} R^{-1}(\mathbf{y}-\boldsymbol{\xi})\right)\right. \\
& \left.-H(\boldsymbol{\xi}) \exp \left(-\frac{1}{2(\tau-u)}(\mathbf{y}-\boldsymbol{\eta})^{T} R^{-1}(\mathbf{y}-\boldsymbol{\eta})\right)\right]
\end{aligned}
$$

We take $\boldsymbol{\xi}$ to be the dummy spatial vector and $u$ to be the dummy time variable in the Green function. Here, $\boldsymbol{\eta}$ is to be determined in terms of $\boldsymbol{\xi}$ such that the homogeneous boundary condition at $x_{n}=0$ is satisfied. The second term in (7) can be considered as the contribution from the negative source at $\boldsymbol{\eta}$, which is the image of $\boldsymbol{\xi}$ with respect to the boundary mirror $x_{n}=0$. The multiple $H(\boldsymbol{\xi})$ can be considered as the source strength of the image source at $\boldsymbol{\eta}$. The present approach of finding the Green function is called the method of images. The above Green function is easily seen to satisfy the differential equation. It can be shown that $\eta_{1}, \eta_{2}, \ldots, \eta_{n}$ are obtained by solving the following system of algebraic equations (see Appendix B):

$$
\left\{\begin{array}{l}
\boldsymbol{\xi}^{T} R^{-1} \boldsymbol{\xi}=\boldsymbol{\eta}^{T} R^{-1} \boldsymbol{\eta} \\
\mathbf{e}_{i}^{T} R^{-1} \boldsymbol{\xi}=\mathbf{e}_{i}^{T} R^{-1} \boldsymbol{\eta}, \quad i=1,2, \ldots, m,
\end{array}\right.
$$

where $\mathbf{e}_{i}$ is the $i$ th coordinate vector. The solution to the above algebraic system is found to be

$$
\boldsymbol{\eta}=\boldsymbol{\xi}-2 \xi_{n} R \mathbf{e}_{n}
$$

The corresponding value for $H(\boldsymbol{\xi})$ is found to be (see Eq. (B.4) in Appendix B)

$$
H(\boldsymbol{\xi})=\exp \left(\mu_{n} \mathbf{e}_{n}^{T} R^{-1}(\boldsymbol{\xi}-\boldsymbol{\eta})\right)=\exp \left(2 \mu_{n} \xi_{n}\right) .
$$

The Green function can be expressed as

$$
\begin{aligned}
& G_{n}(\mathbf{y}, \tau-u ; \boldsymbol{\xi}) \\
& =\frac{e^{-r(\tau-u)}}{[2 \pi(\tau-u)]^{n / 2}} \frac{1}{\sqrt{\operatorname{det} R}}\left[\exp \left(-\frac{1}{2(\tau-u)}(\mathbf{y}-\boldsymbol{\xi})^{T} R^{-1}(\mathbf{y}-\boldsymbol{\xi})\right)-\exp \left(2 \mu_{n} \xi_{n}\right)\right. \\
& \left.\quad \times \exp \left(-\frac{1}{2(\tau-u)}\left(\mathbf{y}+2 \xi_{n} R \mathbf{e}_{n}-\boldsymbol{\xi}\right)^{T} R^{-1}\left(\mathbf{y}+2 \xi_{n} R \mathbf{e}_{n}-\boldsymbol{\xi}\right)\right)\right]
\end{aligned}
$$

The above Green function is valid for external barrier which is either downstream or upstream. To facilitate the subsequent derivation of the option formulas, it is more convenient to use an alternative form of the Green function as given by Eq. (C.3) in Appendix C. For convenience, we take the dummy time variable $u$ to be zero in all subsequent calculations. 
We restrict the later discussion to options where the number of underlying assets is either one or two. The generalization to arbitrary number of underlying assets is quite straightforward.

\subsection{Barrier options on one underlying asset}

We consider the valuation of European out-options on one underlying asset and with single external barrier. Let $\theta$ denote a binary variable which equals 1 or -1 depending on respectively whether the barrier is downstream or upstream. The interval of definition for $\xi_{2}$ is $(0, \infty)$ or $(-\infty, 0)$ corresponding to $\theta=1$ or -1 , respectively. Also, let $\eta$ be a binary variable which equals 1 or -1 corresponding to the option being a call or a put, respectively. The terminal payoff for any type of option, either a call or a put, can be represented by $\max \left(\eta\left(S_{1, T}-X\right), 0\right)$. Here, $X$ is the strike price and $S_{1, T}$ is the asset price at expiry. The price of an out-option with an external barrier is given by

$$
\begin{aligned}
V & \left(S_{1}, B, \tau\right) \\
= & \int_{0}^{\infty} \int_{\frac{\eta}{\sigma_{1}} \ln X}^{\infty} \eta\left(S_{1, T}-X\right) G_{2}\left(x_{1}, x_{2}, \tau ; \xi_{1}, \xi_{2}\right) d\left(\eta \xi_{1}\right) d\left(\theta \xi_{2}\right) \\
= & \eta S_{1} e^{-q_{1} \tau}\left[N_{2}\left(\eta \widehat{d}_{1},-\theta \widehat{e}_{1} ; \eta \theta \rho_{12}\right)-\exp \left(-2\left(\mu_{2}+\rho_{12} \sigma_{1}\right) x_{2}\right) N_{2}\left(\eta \widehat{d}_{1},-\theta \widehat{e}_{1}^{\prime} ; \eta \theta \rho_{12}\right)\right] \\
& -\eta X e^{-r \tau}\left[N_{2}\left(\eta \widehat{d}_{2},-\theta \widehat{e}_{2} ; \eta \theta \rho_{12}\right)-\exp \left(-2 \mu_{2} x_{2}\right) N_{2}\left(\eta \widehat{d}_{2}^{\prime},-\theta \widehat{e}_{2}^{\prime} ; \eta \theta \rho_{12}\right)\right],
\end{aligned}
$$

where

$$
\begin{aligned}
& \widehat{d}_{2}=\frac{\ln \frac{S_{1}}{X}+\mu_{1} \sigma_{1} \tau}{\sigma_{1} \sqrt{\tau}}, \quad \widehat{d}_{1}=\widehat{d}_{2}+\sigma_{1} \sqrt{\tau}, \quad \widehat{d}_{1}^{\prime}=\widehat{d}_{1}-\frac{2 \rho_{12} x_{2}}{\sqrt{\tau}}, \quad \widehat{d}_{2}^{\prime}=\widehat{d}_{2}-\frac{2 \rho_{12} x_{2}}{\sqrt{\tau}} \\
& \widehat{e}_{1}=-\frac{x_{2}+\left(\mu_{2}+\rho_{12} \sigma_{1}\right) \tau}{\sqrt{\tau}}, \quad \widehat{e}_{2}=-\frac{x_{2}+\mu_{2} \tau}{\sqrt{\tau}}, \quad \widehat{e}_{1}^{\prime}=\widehat{e}_{1}+\frac{2 x_{2}}{\sqrt{\tau}}, \quad \widehat{e}_{2}^{\prime}=\widehat{e}_{2}+\frac{2 x_{2}}{\sqrt{\tau}} .
\end{aligned}
$$

A few corrections have been made to the formula given by Heynen and Kat [1].

\subsection{Options on the extremum of two risky assets with an external barrier}

As an illustration, we consider the valuation of a European call option on the maximum of two risky assets and with a down-and-out external barrier. The payoff function of the above European option is given by $c_{\max }^{2}\left(S_{1}, S_{2}, B, 0\right)=$ $\max \left(\max \left(S_{1, T}, S_{2, T}\right)-X, 0\right)$, where $X$ is the strike price and $S_{i, T}$ is the price of asset $i$ at expiry, $i=1,2$. The option remains alive only when $B>b(\tau)=b_{0} e^{-\beta \tau}$ throughout the whole life of the option. The background on the use of options on the extremum of several risky assets can be found in Stulz's paper [7]. In what follows, we adopt similar derivation procedure proposed by Johnson [2]. 
Formally speaking, the price of the present European call is given by

$$
\begin{aligned}
c_{\max }^{2}\left(S_{1}, S_{2}, B, \tau\right)= & \int_{0}^{\infty} \int_{-\infty}^{\infty} \int_{-\infty}^{\infty} c_{\max }^{2}\left(S_{1}, S_{2}, B, 0\right) \\
& G_{3}\left(x_{1}, x_{2}, x_{3}, \tau ; \xi_{1}, \xi_{2}, \xi_{3}\right) d \xi_{1} d \xi_{2} d \xi_{3} .
\end{aligned}
$$

Like all other price formulas, the above call price contains the term which represents the discounted expectation of the cash paid out conditional on the exercising of the call. This cash payment is given by

$$
\begin{aligned}
-X & {\left[\int_{0}^{\infty} \int_{-\infty}^{\infty} \int_{-\infty}^{\infty} G_{3}\left(x_{1}, x_{2}, x_{3}, \tau ; \xi_{1}, \xi_{2}, \xi_{3}\right) d \xi_{1} d \xi_{2} d \xi_{3}\right.} \\
& \left.-\int_{0}^{\infty} \int_{-\infty}^{\frac{1}{\sigma_{2}} \ln X} \int_{-\infty}^{\frac{1}{\sigma_{1}} \ln X} G_{3}\left(x_{1}, x_{2}, x_{3}, \tau ; \xi_{1}, \xi_{2}, \xi_{3}\right) d \xi_{1} d \xi_{2} d \xi_{3}\right] .
\end{aligned}
$$

By defining

$$
u_{i}=\frac{\xi_{i}-y_{i}}{\sqrt{\tau}}, v_{i}=\frac{\xi_{i}-y_{i}+2 \rho_{i 3} x_{3}}{\sqrt{\tau}}, i=1,2,3,
$$

the above second integral term can be expressed as

$$
\begin{aligned}
& e^{-r \tau}\left[\int_{-\frac{y_{3}}{\sqrt{\tau}}}^{\infty} \int_{-\infty}^{\left(\frac{\ln X}{\sigma_{2}}-y_{2}\right) / \sqrt{\tau}} \int_{-\infty}^{\left(\frac{\ln X}{\sigma_{1}}-y_{1}\right) / \sqrt{\tau}} n_{3}\left(u_{1}, u_{2}, u_{3} ; \rho_{12}, \rho_{13}, \rho_{23}\right) d u_{1} d u_{2} d u_{3}\right. \\
& \quad-e^{-2 \mu_{3} x_{3}} \int_{\frac{-y_{3}+2 x_{3}}{\sqrt{\tau}}}^{\infty} \int_{-\infty}^{\left(\frac{\ln X}{\sigma_{2}}-y_{2}+2 \rho_{23} x_{3}\right) / \sqrt{\tau}} \int_{-\infty}^{\left(\frac{\ln X}{\sigma_{1}}-y_{1}+2 \rho_{13} x_{3}\right) / \sqrt{\tau}} \\
& \left.\quad n_{3}\left(v_{1}, v_{2}, v_{3} ; \rho_{12}, \rho_{13}, \rho_{23}\right) d v_{1} d v_{2} d v_{3}\right] \\
& =e^{-r \tau}\left[N_{3}\left(-d,-e, f ; \rho_{12},-\rho_{13},-\rho_{23}\right)\right. \\
& \left.\quad-\exp \left(-2 \mu_{3} x_{3}\right) N_{3}\left(-d^{\prime},-e^{\prime}, f^{\prime} ; \rho_{12},-\rho_{13},-\rho_{23}\right)\right]
\end{aligned}
$$

where

$$
\begin{gathered}
d=\frac{\ln \frac{S_{1}}{X}+\mu_{1} \sigma_{1} \tau}{\sigma_{1} \sqrt{\tau}}, \quad e=\frac{\ln \frac{S_{2}}{X}+\mu_{2} \sigma_{2} \tau}{\sigma_{2} \sqrt{\tau}}, \quad f=\frac{\ln \frac{B}{b(\tau)}+\mu_{3} \sigma_{3} \tau}{\sigma_{3} \sqrt{\tau}} \\
d^{\prime}=d-\frac{2 \rho_{13} \ln \frac{B}{b(\tau)}}{\sigma_{3} \sqrt{\tau}}, \quad e^{\prime}=e-\frac{2 \rho_{23} \ln \frac{B}{b(\tau)}}{\sigma_{3} \sqrt{\tau}}, \quad f^{\prime}=\frac{-\ln \frac{B}{b(\tau)}+\mu_{3} \sigma_{3} \tau}{\sigma_{3} \sqrt{\tau}}
\end{gathered}
$$

Using similar procedure, the evaluation of the first integral gives

$$
e^{-r \tau}\left[N(f)-\exp \left(-2 \mu_{3} x_{3}\right) N\left(f^{\prime}\right)\right] .
$$


Suppose $S_{1, T}$ is the larger of the two asset prices at expiry. Following the procedure proposed by Johnson, we define

$$
\tilde{S}_{1}=\frac{X e^{-r \tau}}{S_{1} e^{-q_{1} \tau}}, \quad \tilde{S}_{2}=\frac{S_{2} e^{-q_{2} \tau}}{S_{1} e^{-q_{1} \tau}}, \quad \tilde{S}_{3}=\frac{B}{b(\tau)},
$$

and let $\tilde{\sigma}_{i}^{2}$ to denote the variance rate of the normal diffusion process for $\ln \tilde{S}_{i}, i=$ $1,2,3$. These variance rates are found to be

$$
\tilde{\sigma}_{1}^{2}=\sigma_{1}^{2}, \quad \tilde{\sigma}_{2}^{2}=\sigma_{1}^{2}-2 \rho_{12} \sigma_{1} \sigma_{2}+\sigma_{2}^{2}, \quad \tilde{\sigma}_{3}^{2}=\sigma_{3}^{2} .
$$

Let $\tilde{\rho}_{i j}$ denote the correlation coefficient between the Wiener processes associated with the logorithms of $\tilde{S}_{i}$ and $\tilde{S}_{j}, i \neq j$. It can be shown that

$$
\tilde{\rho}_{12}=\frac{\sigma_{1}-\rho_{12} \sigma_{2}}{\tilde{\sigma}_{2}}, \quad \tilde{\rho}_{13}=-\rho_{13}, \quad \tilde{\rho}_{23}=\frac{\sigma_{2} \rho_{23}-\sigma_{1} \rho_{13}}{\tilde{\sigma}_{2}} .
$$

Similar to the probability calculation given in (16), the expectation of $S_{1}$ in the risk neutral world, conditional on $\tilde{S}_{1}<1, \tilde{S}_{2}<1$ at expiry and $\tilde{S}_{3}>1$ for all $\tau$, is found to be

$$
\begin{aligned}
& S_{1} e^{-q_{1} \tau}\left[N_{3}\left(d_{1}, e_{1}, f_{1} ; \tilde{\rho}_{12},-\tilde{\rho}_{13},-\tilde{\rho}_{23}\right)\right. \\
& \left.\quad-\exp \left(-2\left(\mu_{3}-\tilde{\rho}_{13} \sigma_{1}\right) x_{3}\right) N_{3}\left(d_{1}^{\prime}, e_{1}^{\prime}, f_{1}^{\prime} ; \tilde{\rho}_{12},-\tilde{\rho}_{13},-\tilde{\rho}_{23}\right)\right],
\end{aligned}
$$

where

$$
\begin{aligned}
& d_{1}=\frac{\ln \frac{S_{1}}{X}+\left(r-q_{1}+\frac{\sigma_{1}^{2}}{2}\right) \tau}{\sigma_{1} \sqrt{\tau}}, \quad e_{1}=\frac{\ln \frac{S_{1}}{S_{2}}+\left(q_{2}-q_{1}+\frac{\widetilde{\sigma_{2}^{2}}}{2}\right) \tau}{\tilde{\sigma}_{2} \sqrt{\tau}} \\
& f_{1}=\frac{\ln \frac{B}{b(\tau)}+\left(\mu_{3} \sigma_{3}-\tilde{\rho}_{13} \sigma_{1} \sigma_{3}\right) \tau}{\sigma_{3} \sqrt{\tau}} \\
& d_{1}^{\prime}=d_{1}+\frac{2 \tilde{\rho}_{13} \ln \frac{B}{b(\tau)}}{\sigma_{3} \sqrt{\tau}}, \quad e_{1}^{\prime}=e_{1}+\frac{2 \tilde{\rho}_{23} \ln \frac{B}{b(\tau)}}{\sigma_{3} \sqrt{\tau}} \\
& f_{1}^{\prime}=\frac{-\ln \frac{B}{b(\tau)}+\left(\mu_{3} \sigma_{3}-\widetilde{\rho}_{13} \sigma_{1} \sigma_{3}\right) \tau}{\sigma_{3} \sqrt{\tau}}
\end{aligned}
$$

Next, suppose $S_{2, T}$ is the larger of the two asset prices at expiry. Accordingly, we define

$$
\hat{S}_{1}=\frac{S_{1} e^{-q_{1} \tau}}{S_{2} e^{-q_{2} \tau}}, \quad \hat{S}_{2}=\frac{X e^{-r \tau}}{S_{2} e^{-q_{2} \tau}}, \quad \hat{S}_{3}=\frac{B}{b(\tau)} .
$$

The variance rates for $\ln \hat{S}_{i}, i=1,2,3$, are found to be

$$
\hat{\sigma}_{1}^{2}=\sigma_{1}^{2}-2 \rho_{12} \sigma_{1} \sigma_{2}+\sigma_{2}^{2}, \quad \hat{\sigma}_{2}^{2}=\sigma_{2}^{2}, \quad \hat{\sigma}_{3}^{2}=\sigma_{3}^{2},
$$


and the corresponding correlation coefficients between $\ln \hat{S}_{i}$ and $\ln \hat{S}_{j}, i \neq j$, are given by

$$
\hat{\rho}_{12}=\frac{\sigma_{2}-\rho_{12} \sigma_{1}}{\hat{\sigma}_{1}}, \quad \hat{\rho}_{13}=\frac{\sigma_{1} \rho_{13}-\sigma_{2} \rho_{23}}{\hat{\sigma}_{1}}, \quad \hat{\rho}_{23}=-\rho_{23} .
$$

The expectation of $S_{2}$ in the risk neutral world, conditional on $\hat{S}_{1}<1, \hat{S}_{2}<1$ at expiry and $\hat{S}_{3}>1$ for all $\tau$, is found to be

$$
\begin{aligned}
& S_{2} e^{-q_{2} \tau}\left[N_{3}\left(d_{2}, e_{2}, f_{2} ; \hat{\rho}_{12},-\hat{\rho}_{13},-\hat{\rho}_{23}\right)\right. \\
& \left.\quad-\exp \left(-2\left(\mu_{3}-\hat{\rho}_{23} \sigma_{2}\right) x_{3}\right) N_{3}\left(d_{2}^{\prime}, e_{2}^{\prime}, f_{2}^{\prime} ; \hat{\rho}_{12},-\hat{\rho}_{13},-\hat{\rho}_{23}\right)\right]
\end{aligned}
$$

where

$$
\begin{gathered}
d_{2}=\frac{\ln \frac{S_{2}}{S_{1}}+\left(q_{1}-q_{2}+\frac{\hat{\sigma}_{1}^{2}}{2}\right) \tau}{\hat{\sigma}_{1} \sqrt{\tau}}, \quad e_{2}=\frac{\ln \frac{S_{2}}{X}+\left(r-q_{2}+\frac{\sigma_{2}^{2}}{2}\right) \tau}{\sigma_{2} \sqrt{\tau}}, \\
f_{2}=\frac{\ln \frac{B}{b(\tau)}+\left(\mu_{3} \sigma_{3}-\hat{\rho}_{23} \sigma_{2} \sigma_{3}\right) \tau}{\sigma_{3} \sqrt{\tau}}, \quad d_{2}^{\prime}=d_{2}+\frac{2 \hat{\rho}_{13} x_{3}}{\sqrt{\tau}} \\
e_{2}^{\prime}=e_{2}+\frac{2 \hat{\rho}_{23} x_{3}}{\sqrt{\tau}}, \quad f_{2}^{\prime}=\frac{-\ln \frac{B}{b(\tau)}+\left(\mu_{3} \sigma_{3}-\hat{\rho}_{23} \sigma_{2} \sigma_{3}\right) \tau}{\sigma_{3} \sqrt{\tau}}
\end{gathered}
$$

Combining the results, the price of the European call option on the maximum of two risky assets is given by

$$
\begin{aligned}
c_{\max }^{2}( & \left.S_{1}, S_{2}, B, \tau\right) \\
= & S_{1} e^{-q_{1} \tau}\left[N_{3}\left(d_{1}, e_{1}, f_{1} ; \tilde{\rho}_{12},-\tilde{\rho}_{13},-\tilde{\rho}_{23}\right)-\exp \left(-2\left(\mu_{3}-\tilde{\rho}_{13} \sigma_{1}\right) x_{3}\right)\right. \\
& \times N_{3}\left(d_{1}^{\prime}, e_{1}^{\prime}, f_{1}^{\prime} ; \tilde{\rho}_{12},-\tilde{\rho}_{13},-\tilde{\rho}_{23}\right)+S_{2} e^{-q_{2} \tau}\left[N_{3}\left(d_{2}, e_{2}, f_{2} ; \hat{\rho}_{12},-\hat{\rho}_{13},-\hat{\rho}_{23}\right)\right. \\
& \left.-\exp \left(-2\left(\mu_{3}-\hat{\rho}_{23} \sigma_{2}\right) x_{3}\right) N_{3}\left(d_{2}^{\prime}, e_{2}^{\prime}, f_{2}^{\prime} ; \hat{\rho}_{12},-\hat{\rho}_{13},-\hat{\rho}_{23}\right)\right] \\
& -X e^{-r \tau}\left\{\left[N(f)-N_{3}\left(-d,-e, f ; \rho_{12},-\rho_{13},-\rho_{23}\right)\right]\right. \\
& \left.-\exp \left(-2 \mu_{3} x_{3}\right)\left[N\left(f^{\prime}\right)-N_{3}\left(-d^{\prime},-e^{\prime}, f^{\prime} ; \rho_{12},-\rho_{13},-\rho_{23}\right)\right]\right\} .
\end{aligned}
$$

As a verification, suppose we set $S_{2}=0$ in (26) so that

$$
\begin{aligned}
c_{\max }^{2}( & \left.S_{1}, 0, B, \tau\right) \\
= & S_{1} e^{-q_{1} \tau}\left[N_{3}\left(d_{1}, \infty, f_{1} ; 1, \rho_{13}, \rho_{13}\right)-\exp \left(-2\left(\mu_{3}+\rho_{13} \sigma_{1}\right) x_{3}\right)\right. \\
& \left.\times N_{3}\left(d_{1}^{\prime}, \infty, f_{1}^{\prime} ; 1, \rho_{13}, \rho_{13}\right)\right]-X e^{-r \tau}\left[N(f)-N_{3}\left(-d, \infty, f ; 0,-\rho_{13}, 0\right)\right. \\
& -\exp \left(-2 \mu_{3} x_{3}\right)\left[N\left(f^{\prime}\right)-N_{3}\left(-d^{\prime}, \infty, f^{\prime} ; 0,-\rho_{13}, 0\right)\right] \\
= & S_{1} e^{-q_{1} \tau}\left[N_{2}\left(d_{1}, f_{1} ; \rho_{13}\right)-\exp \left(-2\left(\mu_{3}+\rho_{13} \sigma_{1}\right) x_{3}\right) N_{2}\left(d_{1}^{\prime}, f_{1}^{\prime} ; \rho_{13}\right)\right] \\
& -X e^{-r \tau}\left[N_{2}\left(d, f ; \rho_{13}\right)-\exp \left(-2 \mu_{3} x_{3}\right) N_{2}\left(d^{\prime}, f^{\prime} ; \rho_{13}\right)\right] .
\end{aligned}
$$


The above price formula agrees with that given in (12) with $\theta=\eta=1$, as expected.

The prices of other European options on the extremum of several risky assets and with an external barrier can be derived in a similar manner.

\section{Numerical Algorithms}

It has been fortunate that analytic price formulas are available for the above European options with an external barrier. However, in most other option models with similar nature, the corresponding price formula may appear to be too cumbersome or unavailable. A good example is the single-asset European option with both internal (dependent on the underlying asset price) and external barriers, whose analytic price formula involves an infinite series since infinite number of images will be generated based on the present framework of method of images. Also, no analytic price formula would be available for multi-asset options when the terminal payoff function is a linear function of the underlying asset prices. In these cases, one has to resort to numerical method for valuation of the option models.

The popular numerical approaches are the binomial method and the finite difference method. The binomial method may not be a good choice for the present external barrier option models since it is well known to have difficulty to cope with the presence of barriers [6]. In this paper, we propose a class of fractional step finite difference schemes for the numerical valuation of European options with an external barrier. Fractional splitting technique has been commonly adopted in numerical algorithms for fluid flow simulation [4]. The scheme proposed below can be considered as the fractional splitting of the second order time accurate Crank-Nicholson scheme. The advancement in each time step is split into $n$ stages, where $n$ is the number of spatial dimensions in the governing equation. In every stage, only the implicit differencing of spatial derivative terms in one of the spatial variables (one by one in turn) is involved; and so only the solution of a tridiagonal system of equations is required at each stage. This splitting approach avoids the solution of a linear system at every time step required by the full form of the Crank-Nicholson scheme. The fractional step procedure can be considered as performing one Gauss-Seidel relaxation in each spatial direction, taken consecutively. If desired, the relaxation procedure in each direction may be repeated to achieve higher level of accuracy.

\subsection{Single-asset option with an external barrier}

We illustrate the splitting procedure through the valuation of the following option model: a European call option on a single asset with an external down-andout barrier and no rebate. The governing equation of the call is given by

$$
\begin{aligned}
\frac{\partial c}{\partial \tau}= & \frac{1}{2}\left(\frac{\partial^{2} c}{\partial x_{1}^{2}}+2 \rho_{12} \frac{\partial c^{2}}{\partial x_{1} \partial x_{2}}+\frac{\partial^{2} c}{\partial x_{2}^{2}}\right)+\mu_{1} \frac{\partial c}{\partial x_{1}}+\mu_{2} \frac{\partial c}{\partial x_{2}}-r c, \\
& -\infty<x_{1}<\infty, \quad 0<x_{2}<\infty, \quad \tau>0
\end{aligned}
$$


where

$$
x_{1}=\frac{1}{\sigma_{1}} \ln S, \quad x_{2}=\frac{1}{\sigma_{2}} \ln \frac{B}{b(\tau)}, \quad b(\tau)=b_{0} e^{-\beta \tau}, \quad \beta>0 .
$$

The boundary conditions at $S=0$ and $B=b(\tau)$ can be deduced to be

$$
\lim _{x_{1} \rightarrow-\infty} c\left(x_{1}, x_{2}, \tau\right)=0 \quad \text { and } \quad c\left(x_{1}, 0, \tau\right)=0
$$

When $B$ attains sufficiently large value, it becomes much unlikely that the downand-out barrier is breached. Hence, the far field boundary condition at large value of $B$ is

$$
\lim _{x_{2} \rightarrow \infty} c\left(x_{1}, x_{2}, \tau\right)=c_{E}\left(x_{1}, \tau\right)
$$

where $c_{E}\left(x_{1}, \tau\right)$ is the price formula for the corresponding European option without the barrier. It is slightly more trickly to deduce the far field boundary condition at large value of $S$. At exceedingly high value of $S$, the option is sure to expire in-themoney, provided that the value of the barrier variable stays above the barrier level for all times. The limiting value of the corresponding vanilla call option at high asset value is $e^{\sigma_{1} x_{1}-q_{1} \tau}-X e^{-r \tau}$. Therefore, we may adopt the following boundary condition:

$$
\begin{aligned}
\lim _{x_{1} \rightarrow \infty} c\left(x_{1}, x_{2}, \tau\right)= & E\left[\left(e^{\sigma_{1} x_{1}-q_{1} \tau}-X e^{-r \tau}\right) \mid x_{2}>0\right] \\
= & S e^{-q_{1} \tau}\left[N\left(\bar{e}_{1}\right)-e^{-2\left(\mu_{2}+\rho_{12} \sigma_{1}\right) x_{2}} N\left(\bar{e}_{2}\right)\right] \\
& -X e^{-r \tau}\left[N\left(\bar{e}_{1}^{\prime}\right)-e^{-2 \mu_{2} x_{2}} N\left(\bar{e}_{2}^{\prime}\right)\right]
\end{aligned}
$$

where

$$
\begin{aligned}
& \bar{e}_{1}=\frac{x_{2}+\left(\mu_{2}+\rho_{12} \sigma_{1}\right) \tau}{\sqrt{\tau}}, \quad \bar{e}_{2}=\bar{e}_{1}-\frac{2 x_{2}}{\sqrt{\tau}} \\
& \bar{e}_{1}^{\prime}=\bar{e}_{1}-\rho_{12} \sigma_{1} \sqrt{\tau}, \quad \bar{e}_{2}^{\prime}=\bar{e}_{2}-\rho_{12} \sigma_{1} \sqrt{\tau}
\end{aligned}
$$

The initial condition is simply the terminal payoff function of the call, namely,

$$
c\left(x_{1}, x_{2}, 0\right)=\max \left(e^{\sigma_{1} x_{1}}-X, 0\right) .
$$

The computational procedures of the fractional step finite difference scheme is outlined in Appendix D.

We test the proposed fractional step finite difference algorithm and compare its performance with the binomial method proposed by Kamrad and Ritchken [3]. The accuracy of the numerical schemes is assessed based on the exact value obtained by the direct evaluation of the analytic formula [see Eq. (12)]. In addition, we examine the order of convergence of the numerical schemes. 


\section{Case (i): barrier variable value is far from the down-and-out barrier}

The parameters of the option model are: $B=1.0, b=0.5, r=0.05, q_{1}=0.02$, $\beta=0, \sigma_{1}=0.1, \sigma_{2}=0.2, \rho_{12}=0.5, X=1.0, \tau=1.0$. We find the option value at varying asset values using the numerical schemes and compare the computed values with the exact values. The root-mean-squared error (RMSE) are obtained by summing the squares of these errors at different asset prices and taking the square root. We attempt to deduce the level of accuracy of the numerical schemes and their rate of convergence by performing the calculations at varying time steps and comparing the RMSE values. The numerical results are tabulated in Table 1. Roughly speaking, we observe that when the number of time steps is doubled, the RMSE is halved in binomial calculations but reduced by a factor of one fourth in finite difference calculations. These observations reveal that the finite difference scheme is second order time accurate while the binomial method is only first order time accurate. The fractional splitting procedure, which corresponds to one iteration in each spatial direction, maintains the second order temporal accuracy of the Crank-Nicholson scheme.

\section{Case (ii): barrier variable value is close to the down-and-out barrier}

The parameters of the option model are the same as that in case (i), except that now the barrier $b=0.9$, which is closer to the barrier variable value $B=1.0$. The numerical results for the RMSE values are also tabulated in Table 1 . We observe that the accuracy of the binomial method cannot be improved with increasing number of time steps when the barrier variable value is close to the down-and-out barrier, but the finite difference scheme is shown to remain second order time accurate. One may observe the slowing down of error reduction when the number of time steps goes from 64 to 128. This may be attributed to the dominance of the error from the boundary condition approximation over the truncation error at this level of step

Table 1. Comparison of the root-mean-squared error values with reference to the binomial scheme and the fractional step finite difference scheme at varying number of time steps.

\begin{tabular}{ccccc}
\hline & \multicolumn{2}{c}{$b_{0}=0.5$} & \multicolumn{2}{c}{$b_{0}=0.9$} \\
\hline $\begin{array}{c}\text { number of } \\
\text { time steps }\end{array}$ & binomial & difference & binomial & $\begin{array}{c}\text { finite } \\
\text { difference }\end{array}$ \\
\hline 4 & $1.16 \times 10^{-3}$ & $9.99 \times 10^{-3}$ & $2.44 \times 10^{-2}$ & $9.49 \times 10^{-3}$ \\
8 & $5.00 \times 10^{-4}$ & $3.21 \times 10^{-3}$ & $2.51 \times 10^{-2}$ & $3.19 \times 10^{-3}$ \\
16 & $2.34 \times 10^{-4}$ & $6.98 \times 10^{-4}$ & $2.93 \times 10^{-2}$ & $7.12 \times 10^{-4}$ \\
32 & $1.19 \times 10^{-4}$ & $1.69 \times 10^{-4}$ & $2.70 \times 10^{-2}$ & $1.71 \times 10^{-4}$ \\
64 & $9.68 \times 10^{-5}$ & $4.18 \times 10^{-5}$ & $2.80 \times 10^{-2}$ & $4.91 \times 10^{-5}$ \\
128 & $4.29 \times 10^{-5}$ & $1.03 \times 10^{-5}$ & $2.47 \times 10^{-2}$ & $3.42 \times 10^{-5}$ \\
\hline
\end{tabular}


size. The boundary condition approximation given in (32) becomes less accurate when the value of the barrier variable comes closer to the knock-out level.

\subsection{Option on the maximum of two risky assets with an external barrier}

In order to verify the validity of the pricing formula for the European call option on the maximum of two risky assets with an external down-and-out barrier (see formula (26)), we compute the option value by both direct evaluation of the pricing formula and applying the fractional step algorithm. The parameter values used in the calculations are: $r=0.05, \sigma_{1}=0.4, \sigma_{2}=0.3, \sigma_{3}=0.2, q_{1}=0.08, q_{2}=$ $0.04, q_{3}=0, \rho_{12}=0.5, \rho_{13}=0.5, \rho_{23}=0.5, X=100, S_{1}=100, S_{2}=$ $100, \tau=1.0$; and two choices of the barrier level are taken, namely $b=60$ and $b=80$. The comparison of the numerical results with varying values of the barrier variable is revealed in Table 2 . It is shown that the numerical accuracy of the finite difference calculations is not much affected by the proximity of the value of the barrier variable to the knock-out barrier, a nice property not shared by the usual binomial calculations for barrier option models.

Table 2. This table compares option values obtained by direct evaluation of the pricing formula (26) and applying the fractional step finite difference algorithm, with reference to the European option on the maximum of two risky assets with an external down-and-out barrier. The number of time steps used in the finite difference calculations is 64 .

\begin{tabular}{|c|c|c|c|c|}
\hline & \multicolumn{2}{|c|}{$b=60$} & \multicolumn{2}{|c|}{$b=80$} \\
\hline$B$ & analytic & $\begin{array}{c}\text { finite } \\
\text { difference }\end{array}$ & analytic & $\begin{array}{c}\text { finite } \\
\text { difference }\end{array}$ \\
\hline 80.5 & 19.399 & 19.386 & 1.102 & 1.026 \\
\hline 81.0 & 19.442 & 19.430 & 2.150 & 2.039 \\
\hline 84.0 & 19.598 & 19.622 & 7.426 & 7.513 \\
\hline 87.0 & 19.831 & 19.723 & 11.248 & 11.308 \\
\hline 90.0 & 19.887 & 19.775 & 13.957 & 14.009 \\
\hline
\end{tabular}

The dependence of the option price function on the correlation coefficients between the two risky assets and the stochastic movement of the barrier variable is also investigated. There are three correlation coefficients in the option model, namely, $\rho_{12}, \rho_{13}$ and $\rho_{23}$. We fix the value of one of the correlation coefficients, $\rho_{12}$, in each figure [see Figs. 1(a), (b) and (c)]. In every figure, three option price curves are shown, where each curve corresponds to one fixed value of the correlation coefficient, $\rho_{23}$. These price curves reveal the dependence of the option price on the correlation coefficient, $\rho_{12}$. In each individual case, the range of $\rho_{12}$ must be chosen such that the correlation coefficient matrix remains positive definite. For 


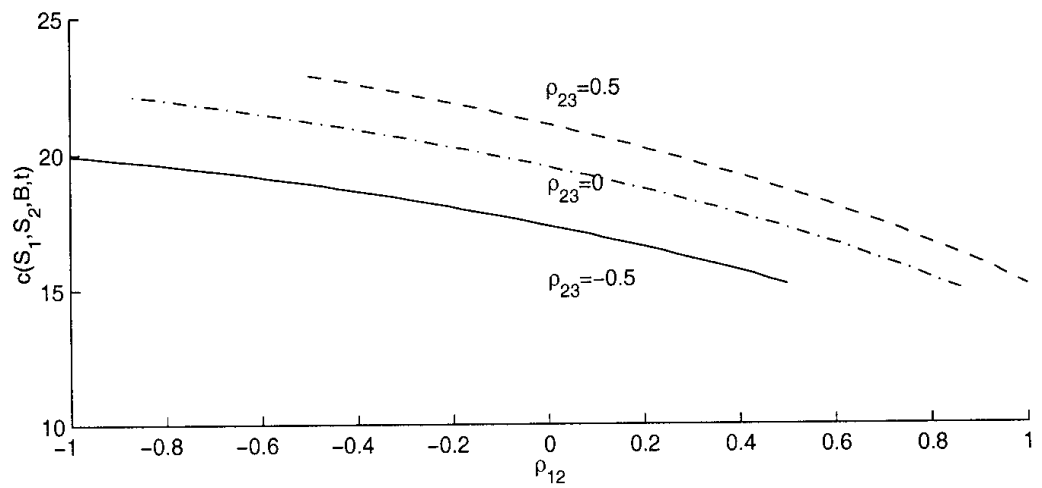

(a)

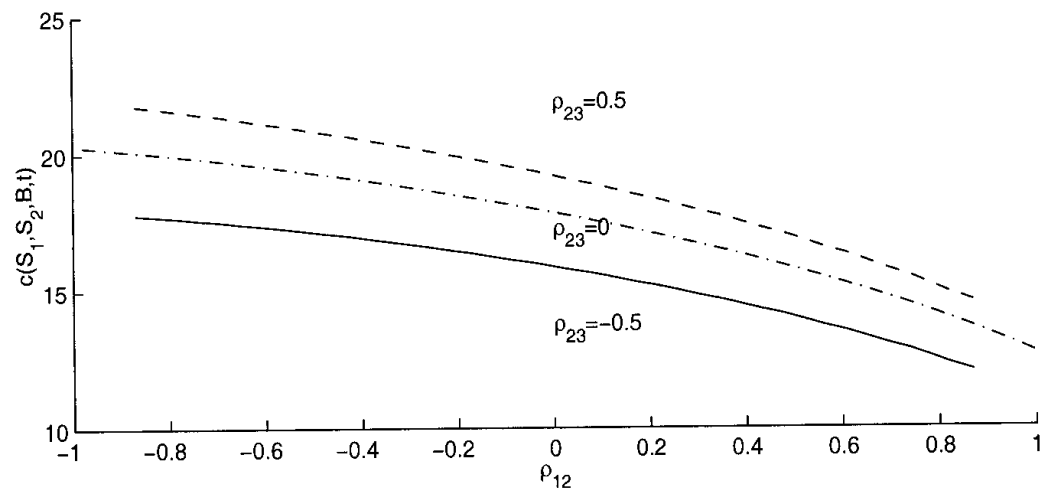

(b)

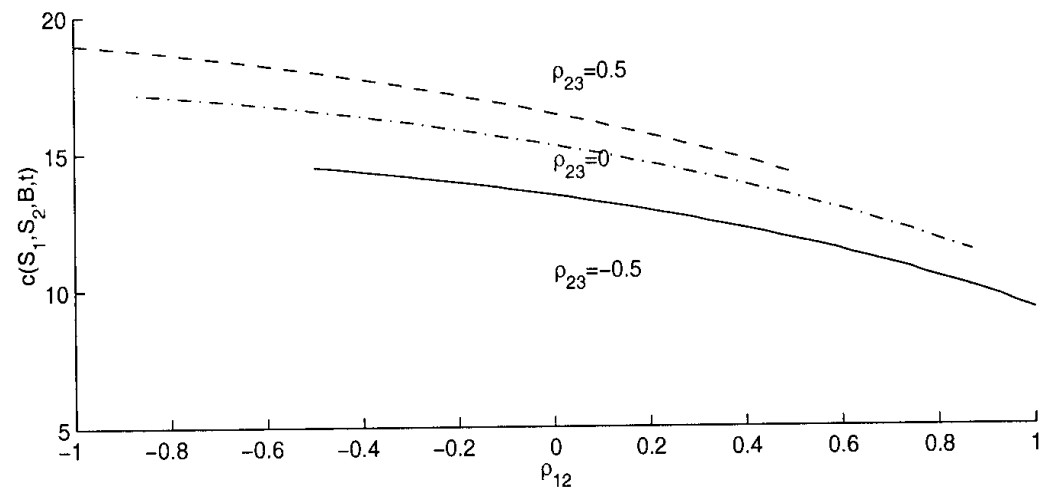

(c)

Fig. 1. Illustration of the dependence of the option price function on the correlation coefficients. The correlation coefficient, $\rho_{13}$, is fixed to be $0.5,0$ and -0.5 in Figs. 1(a), (b) and (c), respectively. In each figure, a set of three values for the correlation coefficient, $\rho_{23}$, is chosen. The dashed curve corresponds to $\rho_{23}=0.5$, the dash-dot curve corresponds to $\rho_{23}=0$, and the solid curve corresponds to $\rho_{23}=-0.5$. Once $\rho_{13}$ and $\rho_{23}$ are fixed, the range of the correlation coefficient, $\rho_{12}$, is chosen so that the correlation coefficient matrix remains positive definite. 
example, with $\rho_{13}=0.5$ [Fig. $\left.1(\mathrm{a})\right]$ and $\rho_{23}=0.5$ (dashed curve), the range for $\rho_{12}$ is taken to be $(-0.5,1)$. The parameter values used in the calculations are: $r=0.05, q_{1}=0.08, q_{2}=0.04, q_{3}=0.1, \sigma_{1}=0.4, \sigma_{2}=0.3, \sigma_{3}=0.2, b=$ $80 e^{-0.1 \tau}, X=100, S_{1}=100, S_{2}=100, B=100, \tau=1.0$.

The plots shown in Fig. 1 reveal that the option price decreases as $\rho_{12}$ increases, but the price increases as either $\rho_{13}$ or $\rho_{23}$ increases. When the correlation coefficient $\rho_{12}$ is negative, the two asset prices tend to move in opposite directions relative to each other. However, since the maximum of the two asset prices is considered, only the higher asset price is taken in calculating the payoff. Even one asset value is moving down, the other asset price is likely moving up, so that high payoff value is secured. The probability of positive terminal payoff would increase with more negative correlation coefficient. Such advantage disappears when the correlation coefficient becomes positive. The asset prices move up or down in pair. Downward moves of both asset prices lead to a drop in payoff value. Hence, the option price is a decreasing function of $\rho_{12}$.

Next, we consider the influences of $\rho_{13}$ or $\rho_{23}$ on the option price. Suppose $\rho_{13}$ is negative, $S_{1}$ and $B$ tend to move in opposite directions. When $S_{1}$ increases, $B$ moves closer to the down-and-out barrier so that the upward increase in option price is limited. On the other hand, when $S_{1}$ decreases, $B$ moves away from the barrier. The payoff decreases while the positive effect of $B$ on option price diminishes, and so option price decreases. The above contradictory effects of $S_{1}$ and $B$ on option price do not occur when $\rho_{13}$ becomes positive. The asset price increases while the barrier variable tends to move away from the barrier. The higher chance of positive terminal payoff is coupled with the lesser chance of being knocked out. Deductively, the option price function is an increasing function of $\rho_{13}$, and also of $\rho_{23}$.

\section{Conclusion}

We have developed a general approach for deriving analytic formulas for the pricing of European multi-asset options with an external barrier. The method of image is shown to be an effective technique for finding the transition density function of the asset price movement with the presence of an external barrier. A second order time accurate fractional step finite difference scheme is proposed. The computational efficiency of the difference scheme is maintained through the splitting procedure. The finite difference scheme is shown to outperform the binomial method, in particular, when the value of the barrier variable is close to the knock-out barrier. Apparently, the accuracy of the finite difference calculations is not much affected by the proximity of the value of the barrier variable to the knock-out barrier. Some pricing behaviors of the European call option on the maximum of two risky assets with an external down-and-out barrier are also discussed. In particular, the dependence of the option price function on the correlation coefficients between the asset price processes and the stochastic movement of the barrier variable is considered. The option price decreases when the correlation between the two risky assets increases, 
but increases when the correlation between the barrier variable and either one of the risky assets increases.

\section{Appendix A}

We would like to derive the fundamental solution of the following $n$-dimensional parabolic equation defined in the infinite domain

$$
\begin{gathered}
\frac{\partial V}{\partial \tau}=\frac{1}{2} \sum_{i=1}^{n} \sum_{j=1}^{n} \rho_{i j} \frac{\partial^{2} V}{\partial x_{i} \partial x_{j}}+\sum_{i=1}^{n} \mu_{i} \frac{\partial V}{\partial x_{i}}-r V \\
-\infty<x_{i}<\infty, \quad i=1,2, \ldots, n .
\end{gathered}
$$

Define a new set of variables

$$
y_{i}=x_{i}+\mu_{i} \tau, \quad i=1,2, \ldots, n,
$$

and

$$
\phi=e^{r \tau} V
$$

then the above equation can be transformed into

$$
\begin{aligned}
\frac{\partial \phi}{\partial \tau} & =\frac{1}{2} \sum_{i=1}^{n} \sum_{j=1}^{n} \rho_{i j} \frac{\partial^{2} \phi}{\partial y_{i} \partial y_{j}} \\
& =\frac{1}{2}\left(\frac{\partial}{\partial y_{1}} \frac{\partial}{\partial y_{2}} \cdots \frac{\partial}{\partial y_{n}}\right) R\left(\begin{array}{c}
\frac{\partial}{\partial y_{1}} \\
\frac{\partial}{\partial y_{2}} \\
\vdots \\
\frac{\partial}{\partial y_{n}}
\end{array}\right) \phi, \quad-\infty<y_{i}<\infty,
\end{aligned}
$$

where $R$ is a $n \times n$ symmetric matrix whose entries are $R_{i j}=\rho_{i j}, i, j=1,2, \ldots, n$. The correlation coefficients are specified such that $R$ is positive definite, otherwise, arbitrage opportunities can be derived from the assets and their derivatives. As a consequence, there exists an orthonormal matrix $Q$ such that

$$
Q^{T} R Q=\Lambda
$$

where $\Lambda$ is a diagonal matrix whose entries are the eigenvalues of $R$. When $R$ is positive definite, all eigenvalues of $R$ are positive. We apply the following linear transformation of variables:

$$
\left(z_{1} z_{2} \ldots z_{n}\right)^{T}=\Lambda^{-\frac{1}{2}} Q^{T}\left(\begin{array}{lll}
y_{1} & y_{2} & \ldots y_{n}
\end{array}\right)^{T},
$$


where $\Lambda^{-\frac{1}{2}}$ denotes the inverse of the positive square root of the diagonal matrix $\Lambda$. This leads to the following prototype $n$-dimensional diffusion equation:

$$
\frac{\partial \phi}{\partial \tau}=\frac{1}{2} \sum_{i=1}^{n} \frac{\partial^{2} \phi}{\partial z_{i}^{2}} .
$$

The fundamental solution to (A.6) in the infinite domain is known to be

$$
\begin{aligned}
\phi\left(z_{1}, z_{2}, \ldots, z_{n}, \tau\right)= & \frac{1}{(2 \pi \tau)^{\frac{n}{2}}} \exp \left(-\frac{z_{1}^{2}+z_{2}^{2}+\cdots+z_{n}^{2}}{2 \tau}\right), \\
& -\infty<z_{i}<\infty, i=1,2, \ldots, n
\end{aligned}
$$

Let $\mathbf{y}=\left(\begin{array}{lll}y_{1} & y_{2} \ldots y_{n}\end{array}\right)^{T}$ and observe that $z_{1}^{2}+z_{2}^{2}+\cdots+z_{n}^{2}=\mathbf{y}^{T} R^{-1} \mathbf{y}$ and the Jacobian of the transformation (A.5) is $\frac{1}{\sqrt{\operatorname{det} R}}$. The above fundamental solution can be rewritten as

$$
\begin{aligned}
\phi\left(y_{1}, y_{2}, \ldots, y_{n}, \tau\right)= & \frac{1}{(2 \pi \tau)^{\frac{n}{2}}} \frac{1}{\sqrt{\operatorname{det} R}} \exp \left(-\frac{1}{2 \tau} \mathbf{y}^{T} R^{-1} \mathbf{y}\right), \\
& -\infty<y_{1}, y_{2}, \ldots, y_{n}<\infty
\end{aligned}
$$

\section{Appendix B}

We would like to find $\boldsymbol{\eta}$ in terms of $\boldsymbol{\xi}$ such that $G(\mathbf{y}, \tau-u ; \boldsymbol{\xi})=0$ at $x_{n}=0$. Observe that in general the following quantity

$$
F=\left.\frac{\exp \left(-\frac{1}{2(\tau-u)}(\mathbf{y}-\boldsymbol{\xi})^{T} R^{-1}(\mathbf{y}-\boldsymbol{\xi})\right)}{\exp \left(-\frac{1}{2(\tau-u)}(\mathbf{y}-\boldsymbol{\eta})^{T} R^{-1}(\mathbf{y}-\boldsymbol{\eta})\right)}\right|_{x_{n}=0}
$$

is a function of $\boldsymbol{\xi}$ and $\tau-u$. We determine $\boldsymbol{\eta}$ such that $F$ is a function of $\boldsymbol{\xi}$ only; otherwise, the Green function cannot be expressed in the form given in (7). Now consider

$$
\begin{aligned}
- & \frac{1}{2(\tau-u)}\left[(\mathbf{y}-\boldsymbol{\xi})^{T} R^{-1}(\mathbf{y}-\boldsymbol{\xi})-(\mathbf{y}-\boldsymbol{\eta})^{T} R^{-1}(\mathbf{y}-\boldsymbol{\eta})\right] \\
= & -\frac{1}{2(\tau-u)}\left\{\left(\boldsymbol{\xi}^{T} R^{-1} \boldsymbol{\xi}-\boldsymbol{\eta}^{T} R^{-1} \boldsymbol{\eta}\right)\right. \\
& \left.-2\left[y_{1} \mathbf{e}_{1}^{T} R^{-1}(\boldsymbol{\xi}-\boldsymbol{\eta})+\ldots+y_{n-1} \mathbf{e}_{n-1}^{T} R^{-1}(\boldsymbol{\xi}-\boldsymbol{\eta})+y_{n} \mathbf{e}_{n}^{T} R^{-1}(\boldsymbol{\xi}-\boldsymbol{\eta})\right]\right\},
\end{aligned}
$$

and observe that at $x_{n}=0$, the last term $\frac{1}{\tau-u} y_{n} \mathbf{e}_{n}^{T} R^{-1}(\boldsymbol{\xi}-\boldsymbol{\eta})$ becomes $\mu_{n} \mathbf{e}_{n}^{T} R^{-1}(\boldsymbol{\xi}-\boldsymbol{\eta})$, which is independent of $\tau-u$. Hence, it becomes possible to make $F$ to be independent of $\tau-u$ by choosing $\eta_{1}, \eta_{2}, \ldots, \eta_{n}$ such that

$$
\left\{\begin{array}{l}
\boldsymbol{\xi}^{T} R^{-1} \boldsymbol{\xi}=\boldsymbol{\eta}^{T} R^{-1} \boldsymbol{\eta} \\
\mathbf{e}_{i}^{T} R^{-1}(\boldsymbol{\xi}-\boldsymbol{\eta})=0, \quad i=1,2, \ldots, n-1
\end{array}\right.
$$


One observes that $H(\boldsymbol{\xi})$ is given by $F$ evaluated at $x_{n}=0$. This gives

$$
H(\boldsymbol{\xi})=\exp \left(\mu_{n} \mathbf{e}_{n}^{T} R^{-1}(\boldsymbol{\xi}-\boldsymbol{\eta})\right)=\exp \left(2 \mu_{n} \xi_{n}\right) .
$$

\section{Appendix C}

Consider the last exponential term in (11), we have

$$
\begin{aligned}
(\mathbf{y} & \left.+2 \xi_{n} R \mathbf{e}_{n}-\boldsymbol{\xi}\right)^{T} R^{-1}\left(\mathbf{y}+2 \xi_{n} R \mathbf{e}_{n}-\boldsymbol{\xi}\right), \\
& =\left[\mathbf{y}-\left(I-2 R \mathbf{e}_{n} \mathbf{e}_{n}^{T}\right) \boldsymbol{\xi}\right]^{T} R^{-1}\left[\mathbf{y}-\left(I-2 R \mathbf{e}_{n} \mathbf{e}_{n}^{T}\right) \boldsymbol{\xi}\right] \\
& =\left(A^{-1} \mathbf{y}-\boldsymbol{\xi}\right)^{T} \widehat{R}^{-1}\left(A^{-1} \mathbf{y}-\boldsymbol{\xi}\right),
\end{aligned}
$$

where $A=I-2 R \mathbf{e}_{n} \mathbf{e}_{n}^{T}$ and $\widehat{R}^{-1}=A^{T} R^{-1} A$. Further, let $\mathbf{w}=A^{-1} \mathbf{y}$. For convenience, we may take $u=0$. Now, consider

$$
\begin{aligned}
\exp \left(2 \mu_{n} \xi_{n}\right) \exp \left(-\frac{1}{2 \tau}(\mathbf{w}-\boldsymbol{\xi})^{T} \widehat{R}^{-1}(\mathbf{w}-\boldsymbol{\xi})\right) \\
=\exp \left(-\frac{1}{2 \tau}\left(\mathbf{w}+2 \mu_{n} \tau \widehat{R} \mathbf{e}_{n}-\boldsymbol{\xi}\right)^{T} \widehat{R}^{-1}\left(\mathbf{w}+2 \mu_{n} \tau \widehat{R} \mathbf{e}_{n}-\boldsymbol{\xi}\right)\right) \\
\quad \times \exp \left(2 \mu_{n} \mathbf{e}_{n}^{T} \mathbf{w}\right) \exp \left(2 \mu_{n}^{2} \tau\left(\widehat{R} \mathbf{e}_{n}\right)^{T} \mathbf{e}_{n}\right) \\
=\exp \left(-\frac{1}{2 \tau}(\mathbf{v}-\boldsymbol{\xi})^{T} \widehat{R}^{-1}(\mathbf{v}-\boldsymbol{\xi})\right) \exp \left(-2 \mu_{n} x_{n}\right)
\end{aligned}
$$

where $\mathbf{v}=\mathbf{w}+2 \mu_{n} \tau \widehat{R} \mathbf{e}_{n}$. It can be shown easily that $A^{-1}=A$ and $\widehat{R}^{-1}=R^{-1}$. Hence, the Green function can be expressed in the form

$$
\begin{aligned}
& G_{n}(\mathbf{y}, \tau ; \boldsymbol{\xi}) \\
& =\frac{e^{-r \tau}}{(2 \pi \tau)^{n / 2}} \frac{1}{\sqrt{\operatorname{det} R}}\left[\exp \left(-\frac{1}{2 \tau}(\mathbf{y}-\boldsymbol{\xi})^{T} R^{-1}(\mathbf{y}-\boldsymbol{\xi})\right)\right. \\
& \left.\quad-\exp \left(-2 \mu_{n} x_{n}\right) \exp \left(-\frac{1}{2 \tau}\left(\mathbf{y}-2 x_{n} R \mathbf{e}_{n}-\boldsymbol{\xi}\right)^{T} R^{-1}\left(\mathbf{y}-2 x_{n} R \mathbf{e}_{n}-\boldsymbol{\xi}\right)\right)\right] .
\end{aligned}
$$

Results shown in (11) and (C.3) simply reveal the symmetry property of a Green function. In this new form of the Green function, the multiple function in the second term is in terms of $x_{n}$ rather than $\xi_{n}$. Since the option price formulas are derived based on the spatial integration with respect to $\xi_{1}, \ldots, \xi_{n}$, the new form avoids the tedious procedure of performing completing squares in all subsequent integration. Completing squares is now done once and for all.

\section{Appendix D}

Let $c_{i, j}^{n}$ denote the approximate value of $c\left(i \triangle x_{1}, j \triangle x_{2}, n \triangle \tau\right)$, where $\triangle x_{1}$ and $\triangle x_{2}$ are the stepwidths in $x_{1}$ - and $x_{2}$-directions, respectively, and $\triangle \tau$ is the time 
step. Let $\tilde{c}_{i, j}^{n+1}$ denote the provisional value of $c_{i, j}^{n+1}$ immediate between the $n$th and $(n+1)$ th time steps. Define the centered difference operators as

$$
\delta_{x_{1}}^{2} c_{i, j}=\frac{c_{i+1, j}-2 c_{i, j}+c_{i-1, j}}{\triangle x_{1}^{2}}, \quad D_{x_{1}} c_{i, j}=\frac{c_{i+1, j}-c_{i-1, j}}{2 \triangle x_{1}},
$$

and similar definitions for $\delta_{x_{2}}^{2}$ and $D_{x_{2}}$. The fractional step algorithm is depicted as follows:

(i) provisional stage - implicit differencing of terms involving $x_{1}$-derivatives

$$
\begin{aligned}
\frac{\tilde{c}_{i, j}^{n+1}-c_{i, j}^{n}}{\triangle \tau}= & \frac{1}{2} \delta_{x_{1}}^{2} \frac{\tilde{c}_{i, j}^{n+1}+c_{i, j}^{n}}{2}+\frac{1}{2} \delta_{x_{2}}^{2} c_{i, j}^{n}+\rho_{12} D_{x_{1}} D_{x_{2}} \frac{\tilde{c}_{i, j}^{n+1}+c_{i, j}^{n}}{2} \\
& -\rho_{12} D_{x_{1}} \frac{\tilde{c}_{i, j+1}^{n+1}-c_{i, j+1}^{n}}{2}+\mu_{1} D_{x_{1}} \frac{\tilde{c}_{i, j}^{n+1}+c_{i, j}^{n}}{2}+\mu_{2} D_{x_{2}} c_{i, j}^{n} \\
& -r\left(\frac{\tilde{c}_{i, j}^{n+1}+c_{i, j}^{n}}{2}\right)
\end{aligned}
$$

(ii) final stage - implicit differencing of terms involving $x_{2}$-derivatives

$$
\begin{aligned}
\frac{c_{i, j}^{n+1}-c_{i, j}^{n}}{\triangle \tau}= & \frac{1}{2} \delta_{x_{1}}^{2} \frac{\tilde{c}_{i, j}^{n+1}+c_{i j}^{n}}{2}+\frac{1}{2} \delta_{x_{2}}^{2} \frac{c_{i, j}^{n+1}+c_{i, j}^{n}}{2}+\rho_{12} D_{x_{1}} D_{x_{2}} \frac{c_{i, j}^{n+1}+c_{i, j}^{n}}{2} \\
& -\rho_{12} D_{x_{2}} \frac{c_{i+1, j}^{n+1}-c_{i+1, j}^{n}}{2}+\mu_{1} D_{x_{1}} \frac{\tilde{c}_{i, j}^{n+1}+c_{i, j}^{n}}{2}+\mu_{2} D_{x_{2}} \frac{c_{i, j}^{n+1}+c_{i, j}^{n}}{2} \\
& -r\left(\frac{c_{i, j}^{n+1}+c_{i, j}^{n}}{2}\right),
\end{aligned}
$$

The solution of the tridiagonal system at each stage in the computational procedure can be effected efficiently by the Thomas algorithm.

The extension to higher dimensional multi-asset option models is quite straightforward. For $n$-asset option models, $n$ stages of splitting are required, where each spatial direction is taken in sequence. In each stage, only implicit differencing of terms involving derivatives of one spatial variable is taken. For other derivative terms which are not chosen for implicit treatment, we follow the same spirit as the Gauss-Seidel relaxation where the explicit differencing of average values of known provisional new time level values and old time level values are taken once the provisional values are available.

\section{References}

[1] R. Heynen and H. Kat, Crossing the barrier, Risk 7 (June 1994) 46-51.

[2] H. Johnson, Options on the maximum or the minimum of several assets, J. Financial and Quantitative Analysis 22 (Sept. 1987) 277-283.

[3] B. Kamrad and P. Ritchken, Multinomial approximating models for options with $k$ state variables, Management Science 37(12) (1991) 1640-1652. 
[4] R. Peyret and T. D. Taylor, Computational Methods for Fluid Flow, Springer-Verlag, Berlin (1983).

[5] D. R. Rich, The mathematical foundations of barrier option-pricing theory, Advances in Futures and Options Research 7 (1994) 267-311.

[6] P. Ritchken, On pricing barrier options, J. Derivatives (Winter 1995) 19-28.

[7] R. Stulz, Options on the minimum or the maximum of two risky assets: analysis and applications, J. Financial Economics 10 (July 1982) 161-185. 